CLINICAL STUDY

\title{
Fetal microchimerism in Hashimoto's thyroiditis: a quantitative approach
}

\author{
Michael Klintschar ${ }^{1}$, Uta-Dorothee Immel $^{1}$, Astrid Kehlen ${ }^{2}$, Patrizia Schwaiger ${ }^{3}$, Tarek Mustafa ${ }^{4}$, \\ Sebastian Mannweiler ${ }^{5}$, Sigrid Regauer ${ }^{5}$, Manfred Kleiber ${ }^{1}$ and Cuong Hoang-Vu ${ }^{6}$ \\ Institutes of ${ }^{1}$ Legal Medicine and ${ }^{2}$ Immunology, University Halle-Wittenberg, D06112 Halle/Saale, Germany, ${ }^{3}$ Department of Child and Adolescent \\ Psychiatry, Carl von Basedow Hospital, Merseburg, Germany, ${ }^{4}$ University Clinic of Oral and Maxillo-Facial Surgery, University of Magdeburg, \\ Magdeburg, Germany, ${ }^{5}$ Institute of Pathology, Medical University Graz, A8036 Graz, Austria and ${ }^{6}$ Experimental and Oncological Surgery Research \\ Group, University Department of General, Visceral, and Vascular Surgery Department, University of Halle, Halle, Germany
}

(Correspondence should be addressed to M Klintschar; Email: michael.klintschar@medizin.uni-halle.de)

\begin{abstract}
Objective: Fetal microchimerism (MCH) has been implicated in the etiology of autoimmune diseases such as autoimmune thyroiditis. The goal of the study was to reliably estimate the number of fetal engrafted cells and to further investigate factors influencing the development of MCH.

Methods: Quantitative real-time PCR amplification using Y-chromosome specific (DYS14) and autosomal ( $\beta$-globin) loci was performed on thyroid gland specimens. Furthermore, we compared the distribution of $\mathrm{ABO}$ and rhesus systems in mothers with and without blood $\mathrm{MCH}$ in relation to the blood groups of the children.

Results: $\mathrm{MCH}$ was detected in eight of 21 Hashimoto patients in a frequency range of 15 to 4900 male cells per 100000 total cells (median 97 cells), but in none of 17 healthy thyroid glands. In a third group, consisting of 18 nodular goiters, only one sample was positive (182 male cells/100 000 total cells). No woman who had not had a prior pregnancy with a male fetus showed MCH. Mothers both with and without $\mathrm{MCH}$ showed the same rate of mother/child incompatibilities for the $\mathrm{ABO}$ and rhesus systems.

Conclusions: The percentage of microchimeric cells varies to a great extent in Hashimoto's thyroiditis, and this phenomenon can occur in nodular goiter in rare instances, but it appears to be absent from normal thyroid glands. Nevertheless, the biological significance of $\mathrm{MCH}$ remains unclear. Moreover, we have concluded that the tested blood group systems (as opposed to their role in graft vs host disease after transplantations) have no effect on fetal $\mathrm{MCH}$.
\end{abstract}

European Journal of Endocrinology $154237-241$

\section{Introduction}

The exchange of fetal and maternal cells during pregnancy $(1,2)$ leads to a phenomenon usually referred to as fetal microchimerism ( $\mathrm{MCH})$. Fetal-derived cells have been identified in maternal blood by the unexpected detection of male DNA markers in the maternal circulation and/or maternal tissues as early as 4 weeks of gestation and up to 36 years after pregnancy (3) and may play a role in the development of allogeneic tolerance to the fetus (4).

Up to now only one factor determining the persistence of $\mathrm{MCH}$ is known: the human leukocyte antigen (HLA) compatibility between mother and fetus. This condition resembles that in chronic graft vs host disease with donor lymphocytes reacting with the host tissues. Because chronic graft vs host disease shares many clinical and pathological features with some autoimmune diseases and these kinds of diseases are more common in females past child-bearing years, it has been suggested that fetal MCH might be involved in their etiology (5).

The first disease for which evidence for the involvement of fetal $\mathrm{MCH}$ was found was systemic sclerosis $(4,6)$. Other diseases for which fetal $\mathrm{MCH}$ has been demonstrated (although the clinical significance is still contoversial) are pruritic eruptions of pregnancy (7), primary biliary cirrhosis (8), Sjoegren syndrome $(9,10)$ and autoimmune thyroiditis $(11,12)$. Hashimoto's thyroiditis is one of two major autoimmune thyroid diseases for which a variety of causative agents have been discussed $(13,14)$. We first examined the occurrence of fetal cells in thyroid glands of women suffering from this disease using 
conventional (qualitative) PCR for a Y-chromosomal sequence and demonstrated male cells in eight of 17 patients, but only in one of 25 nodular goiters without evidence of autoimmune disease (11). Fluorescent in situ hybridisation (FISH) analysis using a Y-specific probe confirmed the presence of fetal MCH in Hashimoto's disease (15).

Moreover, fetal $\mathrm{MCH}$ was recently demonstrated for Graves' disease, another thyroid autoimmune disease using ELISA-PCR (12) and a recent study by Renné et al. (15) using FISH confirms the frequent occurrence of male lymphocytes in both autoimmune diseases compared with the rare observation of male cells in adenomas (16).

However, although FISH allows quantitative estimates of the percentage of male cells in a histological slide (Srivatsa et al. (16) report up to 185 male cells per slide), this method is relatively insensitive when compared with real-time PCR (17). We thus performed this procedure on 22 thyroid gland sections from Hashimoto patients, multinodular goiter patients and normal thyroid glands using this robust quantitative approach. In addition, we checked for the presence of fetal blood $\mathrm{MCH}$ in 45 women who had recently given birth to a son and compared these results with rhesus and $\mathrm{ABO}$ blood group types in mother and child to further investigate the factors influencing the persistence of $\mathrm{MCH}$.

\section{Materials and methods}

Fifty-two thyroid gland specimens were collected from the Institute of Pathology of the University of Graz and the Institute of Forensic Medicine of the University of Halle (21 female patients with Hashimoto's thyroiditis, 18 women with multinodular goiter and 17 women with normal thyroid glands (autopsy material)). The diagnosis of Hashimoto's thyroiditis was based on criteria such as destruction of follicular epithelia with diffuse lymphoplasmocytic infiltration, the presence of lymphoid follicles with germinal centers and fibrosis as described (13), whereas multinodular goiters lacked these attributes. Seventeen of the Hashimoto samples and all of the goiter samples had already been used in a previous study (11). DNA was extracted from archival paraffin-embedded thyroid tissue using a commercially available kit (Puregene Kit; Gentra Systems, Minneapolis, MN, USA) comprising a deparaffination step with xylene, proteinase $\mathrm{K}$ digestion, salting out of the proteins and isopropanol precipitation with glycogen as DNA carrier. For each sample, three sections from different layers of the thyroid gland were extracted. DNA quantitation was performed by photometry. DNA extraction from blood was performed as described (18).

Positive controls were DNA from a person in different quantities. Negative controls were one reagent control and one extraction control. Qualitative PCR for the SRY gene was performed as described (11). Real-time quantitative PCR was performed by the use of a Corbett Research Rotorgene 2000 (Corbett Research, Sydney, Australia) which is essentially a combined thermocycler/fluorescence detector with the ability to monitor cycle-for-cycle the success of individual PCR reactions. The amplification and product-reporting system used is based on the $5^{\prime}$ nuclease assay (19). Different from a conventional PCR assay, in a real-time PCR assay not only the two amplification primers but also a dual-labeled fluorigenic hybridization probe is included $(20,21)$. One fluorescent dye (6 carboxyfluorescein (FAM)) serves as a reporter, and its emission spectrum is quenched by a second fluorescent dye (6 carboxy-tetramethylrhodamine (TAMRA)). During the extension phase of PCR, the $5^{\prime}$ to $3^{\prime}$ exonuclease activity of the Taq DNA polymerase cleaves the reporter from the probe, thus releasing it from the quencher, resulting in an increase in fluorescent emission which is detected by the real-time cycler and captured by a computer. The cycle in which a defined threshold is exceeded is used to quantitate the DNA content. In the same run, several standards of known DNA content are used as external standards, and the amount of DNA in the samples is interpolated from the standards. The Y-chromosome-specific (DYS14) assay consisted of the primers 5'-CATCCAGAGCGTCCCTGG-3' and 5'-TTCCCCTTTGTTCCCCAAA-3' and the dual-labeled fluorescent probe $5^{\prime}$-(FAM)CGAAGCCGAGCTGCCCATCA (TAMRA)-3' (22). The $\beta$-globin system consisted of the primers $\beta$-globin-354F, 5'-GTGCACCTGACTCCTGAGGAGA-3' and $\beta$-globin-455R, 5'-CCTTGATACCAACCTGCCCAG-3' and 5'-(FAM)AAGGTGAACGTGGATGAAGTTGGTGG(TAMRA)-3' as probe (23).

Quantitative PCR amplification reactions were set up in a reaction volume of $25 \mu \mathrm{l}$. Each reaction contained $300 \mathrm{nM}$ of each amplification primer, $100 \mathrm{nM}$ of the corresponding probe, $5 \mathrm{mM} \mathrm{MgCl}_{2}, 200 \mathrm{mM}$ each dATP, dCTP, dTTP and dGTP, $25 \mu \mathrm{l} 10 \times$ buffer II and $1.25 \mathrm{U}$ AmpliTaq Gold (Applied Biosystems, Foster City, CA, USA). DNA (10 ng) was used for amplification. Each sample was analyzed three times. Samples which were positive in at least one of the three reactions were amplified another two times. Only positive results in at least two reactions were regarded as positive. The mean of all five reactions was used for all further calculations. The concentration of male DNA, expressed in male DNA copies per total DNA copies was calculated by dividing the results for the DYS14 PCR (= male component) by those for the $\beta$-globin amplification (=total DNA). Extreme caution was taken to avoid false positive results. All tissue samples were handled in the same manner by female technicians. The sections used for DNA extraction were prepared with meticulous care specifically for that purpose and immediately put into Eppendorf tubes. Extraction, PCR assay set-up and 
real-time PCR were performed in separate rooms in separate buildings, and negative controls were always used in every PCR reaction.

\section{Results}

Using a quantitative PCR assay, Y-chromosomal DNA was detected in eight of 21 women suffering from Hashimoto's disease, in one of 18 women suffering from multinodular goiter but in none of 17 healthy thyroid glands (Table 1 ). In the positive samples, the fraction was 15 to 4900 male cells per 100000 total cells (median 97 cells). With the exception of two samples (4900 and 2600 male cells respectively) all tissue samples showed fewer than 250 male cells per 100000 total cells (Fig. 1). The only positive goiter sample showed a rate of 182 male cells/100 000 total cells, being in the range of the Hashimoto samples.

For 14 Hashimoto patients, including seven of the eight patients with $\mathrm{MCH}$, a medical history concerning former pregnancies, transplantations and blood transfusions was available (Table 1). This information was also obtained for the only goiter patient with positive $\mathrm{MCH}$. One patient had had a miscarriage and a blood transfusion in addition to her four children (two boys). Interestingly, although the presence of male cells was only found in females with at least one male child, the percentage of male cells was independent of the number of pregnancies (male or female).

To investigate whether the distribution of blood group systems has an impact on the occurrence of fetal MCH, we typed the rhesus (d, D) and ABO blood groups in 52 mother/son pairs at 6-10 months after delivery of the first son. The presence of male fetal $\mathrm{MCH}$ in the mothers was determined by conventional PCR for the SRY gene as described (11). Mother/son

Table 1 Numbers of sons and daughters of the Hashimoto patients $(\mathrm{H} 1-\mathrm{H} 13)$ for whom a family history was available in relation to the male genomes per 100000 total genomes. The only struma nodosa patient with fetal $\mathrm{MCH}$ (SN1) is displayed in italics.

\begin{tabular}{lccc}
\hline Patient & Sons & Daughters & $\begin{array}{c}\text { Male genomes } \\
\text { (per 100 000 total genomes) }\end{array}$ \\
\hline H1 & 0 & 0 & 0.00 \\
H2 & 0 & 0 & 0.00 \\
H3 & 1 & 0 & 0.00 \\
H4 & 1 & 0 & 0.00 \\
H5 & 1 & 1 & 0.00 \\
H6 & 1 & 0 & 15.37 \\
H7 & 1 & 1 & 87.77 \\
H8 & 1 & 1 & 139.33 \\
H9 & 1 & 0 & 250.91 \\
H10 & 1 & 1 & 4862.49 \\
H11 & 2 & 2 & 23.31 \\
H12 & 2 & 1 & 36.21 \\
SN1 & 2 & 0 & 18.25 \\
H13 & 3 & 1 & 0.00 \\
\hline
\end{tabular}

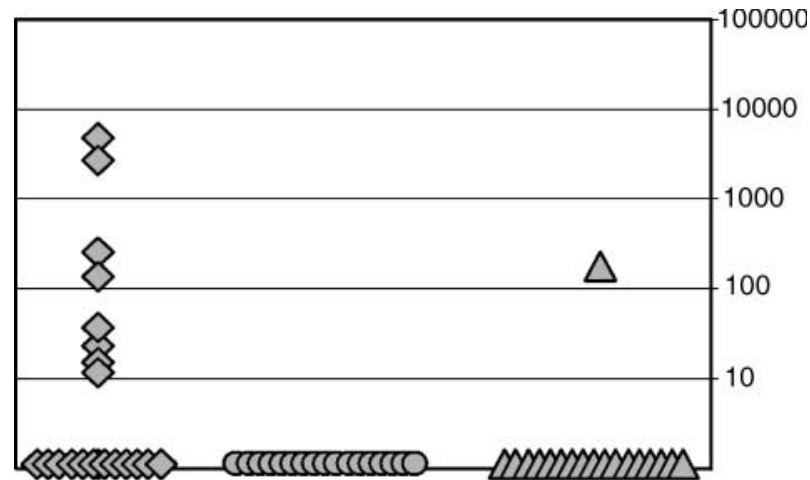

$\diamond$ Hashimoto 0 Normal thyroid $\Delta$ Nodular goiter

Figure 1 Number of male genomes per 100000 female genomes for Hashimoto's thyroiditis patients, nodular goiter patients and normal thyroids.

pairs with compatible rhesus and ABO types from the mother's point of view (e.g. mother D and son D or d) did not show fetal MCH more often than pairs with incompatible types (e.g. mother d and son D) (Table 2).

\section{Discussion}

This is the first study in which fetal $\mathrm{MCH}$ was quantitated in female Hashimoto thyroid specimens and compared with normal thyroid glands and multinodular goiters using quantitative real-time PCR. Although FISH analysis gave estimates of fetal cells per slide in different thyroid diseases in previous studies $(15,16)$, this is a relatively insensitive technique. A study on fetal MCH in systemic sclerosis suggests a superior sensitivity of quantitative real-time PCR compared with FISH (17). In our study, we tried to further increase this sensitivity by using DYS14 (24) as the male index. DYS14 is repeated up to 60 times on the Y-chromosome, which makes it superior to single locus genes like SRY (25) in terms of sensitivity. In theory, only a small fraction of a single male genome per assay can be detected (22).

Moreover, most comparable studies tested only a very small number of samples: e.g. in the study by Ando et al. (12) seven cases of Graves' disease were tested and among the samples studied by Srivatsa et al. (16)

Table 2 Incompatibilities of the child for the ABO and rhesus system (only D) from the perspective of the mother. Healthy mothers for whom a fetal $\mathrm{MCH}$ was positive in blood 6-10 months after delivery of a son compared with those for whom no fetal $\mathrm{MCH}$ was positive.

\begin{tabular}{lcccr}
\hline & Mothers & ABO (\%) & D (\%) & Total (\%) \\
\hline MCH & 15 & $4(26.7)$ & $1(6.7)$ & $5(33.3)$ \\
No MCH & 30 & $7(23.3)$ & $3(10.0)$ & $10(33.3)$ \\
\hline
\end{tabular}


there were only six Hashimoto cases. In the latter study using FISH, the number of male cells per slide ranged between 0 and 185, data which are hard to compare with those presented here by us. However, there are data on the percentage of male cells in another autoimmune disease (Graves' disease) using a similar PCRbased quantitative approach (ELISA-PCR) (12). In this study on seven patients there were up to 295 male cells per 100000 total cells. Our results in a much larger sample of Hashimoto patients appear to suggest that the number of microchimeric cells might be similar in both autoimmune thyroid diseases, although this percentage appears to vary widely.

However, our results and those of Srivatsa et al. (16) differ in the frequency of $\mathrm{MCH}$ in the patients studied. While we found male cells in eight of 21 Hashimoto patients, FISH analysis revealed $\mathrm{MCH}$ in five of six cases. Renné et al. (15) in their FISH analysis performed on a larger sample size found percentages similar to those observed in our study $(60 \%)$. These differences might be explained either by the relatively small sample sizes or by different sensitivities of FISH and real-time PCR. All studies, however, agree on a lack of $\mathrm{MCH}$ in normal thyroid glands.

As male cells were only detected in mothers with at least one son, pregnancy appears to be (in the absence of transplants or transfusions) a condition sine qua non for $\mathrm{MCH}$. However, as the frequency of male fetal cells is independent of the number of male pregnancies, other factors - in analogy with chimerism after organ transplants (4) - appear to determine whether MCH is persistent or not. In that respect, HLA compatibility or incompatibility from the host's (mother) point of view is generally recognized to be the only known determining factor (5). However, as the ABO blood type distribution of graft and host influences the occurrence of chimerism and graft vs host reaction in transplantations $(26,27)$, we reasoned that the distribution of the blood groups in mother and child might be another factor determining the persistence of MCH. Nevertheless, as we found $\mathrm{MCH}$ to be as frequent in mother/son pairs with and without $\mathrm{ABO}$ or rhesus incompatibility (Table 2), we assume that, other than HLA types, blood group systems do not influence the probability of developing persistent fetal $\mathrm{MCH}$. This might be the consequence of a lack of expression of blood group antigens on the microchimeric cells.

The etiologic consequences of fetal $\mathrm{MCH}$ are difficult to assess to date. Of course, the fact that this phenomenen is significantly more common in autoimmune disease compared with normal tissue fosters a potential role of this phenomenon in the pathogenesis of autoimmune thyroid disease $(28,29)$. Nevertheless, up to now only the presence of fetal engrafted cells in thyroid autoimmune disease is proven, but not an actual active, adverse role of $\mathrm{MCH}$ in the autoimmune process. An argument against an active role is that only a part (in most studies roughly $50 \%$ ) of all thyroid autoimmune disease patients show $\mathrm{MCH}(11,12,15,16$, this study). Moreover, a recent study proved that parity is not a risk factor for thyroid autoimmunity (30). To explain the biological significance of this phenomenon it has been speculated that microchimeric cells might, in fact, not be the source of the inflammation, but a reaction of the body to the inflammation (31). As at least some of the engrafted fetal cells have stem cell potential, these cells might be part of a repair process. For thyroid diseases, this hypothesis seems to be supported by the fact that $\mathrm{MCH}$ is also observed in nonautoimmune diseases (15, this study) and for other tissues by the recent observation of $\mathrm{MCH}$ in non-autoimmune diseases of the vagina and uterus $(32,33)$. Moreover, Srivatsa et al. (16) were able to identify the microchimeric cells in at least one patient as normal thyrocytes (and not immuncompetent cells).

In conclusion, this is the first study to quantitate the rate of microchimeric cells using a highly sensitive quantitative PCR approach. In doing so we found male microchimeric cells in the thyroid glands of eight of 21 female Hashimoto patients, varying between 15 and 4900 in 100000 cells. In the nodular goiter control group, only one patient was positive for male cells with a ratio well in the range of the Hashimoto patients (182 in 100000). In normal thyroid glands, on the other hand, no male cells were observed. In addition, the persistence of $\mathrm{MCH}$ appears to be independent of $\mathrm{ABO}$ and rhesus compatibility between mother and fetus.

\section{References}

1 Schroder J. Transplacental passage of blood cells. Journal of Medical Genetics 197512 230-242.

2 Hsieh TT, Pan CC, Hor JJ \& Kao SM. Presence of fetal cells in maternal circulation after delivery. Human Genetics 199392 204-205.

3 Bianchi DW, Williams JM, Sullivan LM, Hanson FW, Klinger KW \& Shuber AP. PCR quantification of fetal cells in maternal blood in normal and aneuploid pregnancies. American Journal of Human Genetics 19976 822-829.

4 Nelson JL, Furst DE, Maloney S, Gooley T, Evans PC, Smith A, Bean MA, Ober C \& Bianchi DW. Microchimerism and HLA-compatible relationships of pregnancy in systemic sclerosis. Lancet $1998351559-562$.

5 Lambert N \& Nelson JL. Microchimerism in autoimmune disease: more questions than answers? Autoimmunity Reviews 20032 133-139.

6 Artlett C, Smith J \& Jimenez S. Identification of fetal cells in skin lesions from women with systemic sclerosis. New England Journal of Medicine 1998338 1186-1191.

7 Aractingi S, Berkane N, Bertheau P, Le Goue C, Dausset J, Uzan S \& Carosella ED. Fetal DNA in skin of polymorphic eruptions of pregnancy. Lancet $1999352898-901$.

8 Tanaka A, Lindor K, Gish R, Batts K, Shiratori Y, Omata M, Nelson JL, Ansari A, Coppel R, Newsome M \& Gershwin ME. Fetal microchimerism alone does not contribute to the induction of primary biliary cirrhosis. Hepatology 199930 833-838.

9 Miyashita Y, Ono M, Ono M \& Ueki H. Y chromosome microchimerism in rheumatic autoimmune disease. Annals of Rheumatic Diseases $200059655-666$. 
10 Toda I, Kuwana M, Tsubota K \& Kawakami Y. Lack of evidence for an increased microchimerism in the circulation of patient with Sjogren's syndrome. Annals of Rheumatic Diseases 200160 248-253.

11 Klintschar Klintschar M, Schwaiger P, Mannweiler S, Regauer S \& Kleiber M. Evidence of fetal microchimerism in Hashimoto's thyroiditis. Journal of Clinical Endocrinology and Metabolism 200186 2494-2498.

12 Ando T, Imaizumi M, Graves PN, Unger P \& Davies TF. Intrathyroidal fetal microchimerism in Graves' disease. Journal of Clinical Endocrinology and Metabolism 200287 3315-3320.

13 Dayan CM \& Daniels GH. Chronic autoimmune thyreoiditis. New England Journal of Medicine 1997335 99-107.

14 Prummel MF, Strieder T \& Wiersinga WM. The environment and autoimmune thyroid diseases. European Journal of Endocrinology $2004150605-618$.

15 Renne C, Ramos Lopez E, Steimle-Grauer SA, Ziolkowski P, Panni MA, Luther C, Holzer K, Encke Albrecht, Wahl RA, Bechstein WO, Usadel KH, Hansmann ML \& Badenhoop K. Thyroid fetal male microchimerisms in mothers with thyroid disorders: presence of Y-chromosomal immunofluorescence in thyroidinfiltrating lymphocytes is more prevalent in Hashimoto's thyroiditis and Graves' disease than in follicular adenomas. Journal of Clinical Endocrinology and Metabolism $2004895810-5814$.

16 Srivatsa B, Srivatsa S, Johnson KI, Samura O, Lee St \& Bianchi DW. Microchimerism of presumed fetal origin in thyroid specimens of women. A case-control study. Lancet 2001358 2034-2038.

17 Sawaya HHB, Jimenez SA \& Artlett CM. Quantification of fetal microchimeric cells in clinically affected and unaffected skin of patients with systemic sclerosis. Rheumatology $2004 \mathbf{4 3}$ 965-968.

18 Klintschar M, Dauber EM, Ricci U, Cerri N, Immel UD, Kleiber M \& Mayr WR. Haplotype studies support slippage as the mechanism of germline mutations in short tandem repeats. Electrophoresis $2004203344-3348$.

19 Holland PM, Abramson RD, Watson R \& Gelfand DH. Detection of specific polymerase chain reaction product by utilizing the $5^{\prime}-3^{\prime}$ exonuclease activity of the thermus aquaticus DNA polymerase. PNAS $1991 \mathbf{8 8} 7276-7280$.

20 Lee LG, Connell CR \& Bloch W. Allelic discrimination by nicktranslation PCR with fluorogenic probes. Nucleic Acids Research $1993213761-3766$.

21 Livak KJ, Flood SJ, Marmaro J, Giusti W \& Deetz K. Oligonucleotides with fluorescent dyes at opposite ends provide a quenched probe system useful for detecting PCR product and nucleic acid hybridization. PCR Methods and Applications 19954 357-362.

22 Lambert NC, Lo YM, Erickson TD, Tylee TS, Guthrie KA, Furst DE \& Nelson JL. Male microchimerism in healthy women and women with scleroderma: cells or circulating DNA? A quantitative answer. Blood $20021002845-2851$.

23 Lo YM, Tein MS, Lau TK, Haines CJ, Leung TN, Poon PM, Wainscoat JS, Johnson PJ, Chang AM \& Hjelm NM. Quantitative analysis of fetal DNA in maternal plasma and serum: implications for noninvasive prenatal diagnosis. American Journal of Human Genetics $1998 \mathbf{6 2} 768-775$.

24 Arnemann J, Epplen JT, Cooke HJ, Sauermann U, Engel W \& Schmidtke J. A human Y-chromosomal DNA sequence expressed in testicular tissue. Nucleic Acids Research 198715 8713-8724.

25 Zhong XY, Burk MR, Troeger C, Kang A, Holzgreve W \& Hahn S. Fluctuation of maternal and fetal free extracellular circulatory DNA in maternal plasma. Obstetrics and Gynecology 200096 991-996.

26 Svennilson J, Remberger M \& Ringden O. Risk factors for moderate to severe acute graft-vs.-host disease after allogeneic stem cell transplantation in children. Pediatric Transplantation 20037 $130-136$.

27 Bogunia-Kubik K, Suchnicki K \& Lange A. HLA-DR11 in addition to donor age, gender, and major blood group incompatibility influence the incidence of acute graft-versus-host disease after allogeneic hematopoietic stem cell transplantation. Transplantation Proceedings 200335 1556-1558.

28 Ando T \& Davies TF. Postpartum autoimmune thyroid disease: the potential role of fetal microchimerism. Journal of Clinical Endocrinology and Metabolism 200388 2965-2971.

29 Badenhoop K. Intrathyroidal microchimerism in Graves' disease or Hashimoto's thyroiditis: regulation of tolerance or alloimmunity by fetal-maternal immune interactions? European Journal of Endocrinology $2004150421-423$.

30 Walsh JP, Bremner AP, Bulsara MK, O'Leary P, Leedman PJ, Feddema P \& Michelangeli V. Parity and the risk of autoimmune thyroid disease: a community-based study. Journal of Clinical Endocrinology and Metabolism 200590 5309-5312.

31 Lee Nelson J. Microchimerism: incidental byproduct of pregnancy or active participant in human health? Trends in Molecular Medicine 20008 109-113.

32 Regauer S, Schwaiger S, Liegl B \& Klintschar M. Fetal microchimerism is common in normal and diseased vulvar skin. Journal of Investigative Dermatology 2004122 1059-1060.

33 Cha D, Khosrotehrani K, Kim Y, Stroh H, Bianchi DW \& Johnson KL. Cervical cancer and microchimerism. Obstetrics and Gynecology 2003102 774-781.

Received 11 August 2005

Accepted 26 October 2005 